\title{
Synchronization dynamics of mobile oscillators in the presence of coupling delays
}

\author{
Gabriela Petrungaro, ${ }^{1,2,3}$ Koichiro Uriu, ${ }^{4, *}$ and Luis G. Morelli ${ }^{1,2,5,+}$ \\ ${ }^{1}$ Instituto de Investigación en Biomedicina de Buenos Aires (IBioBA)-CONICET-Partner Institute of the Max Planck Society, \\ Polo Científico Tecnológico, Godoy Cruz, 2390, C1425FQD, Buenos Aires, Argentina \\ ${ }^{2}$ Departamento de Física, FCEyN UBA, Ciudad Universitaria, 1428 Buenos Aires, Argentina \\ ${ }^{3}$ Institute for Biological Physics, University of Cologne, Zülpicher Strasse 47a, 50674 Köln, Germany \\ ${ }^{4}$ Graduate School of Natural Science and Technology, Kanazawa University, Kakuma-machi, Kanazawa 920-1192, Japan \\ ${ }^{5}$ Max Planck Institute for Molecular Physiology, Department of Systemic Cell Biology, Otto-Hahn-Strasse 11, D-44227 Dortmund, Germany
}

(Received 31 January 2019; published 10 June 2019)

\begin{abstract}
Individual biological oscillators can synchronize to generate a collective rhythm. During vertebrate development, mobile cells exchange signals to synchronize a rhythmic pattern generator that makes the embryonic segments. Previous theoretical works have shown that cell mobility can enhance synchronization of coupled oscillators when signal exchange is instantaneous. However, in vertebrate segmentation, the exchange of signals is thought to comprise delays from signal sending and processing, which could alter the effect of mobility on synchronization. Here, we study synchronization dynamics of mobile phase oscillators in the presence of coupling delays. We find that mobility can speed up synchronization when coupling delays are present. We derive an analytical expression for the characteristic time of synchronization dynamics, which is in very good agreement with numerical simulations. This analytical expression suggests a subdivision of the mobility range into different dynamical regimes and reveals that, with delayed coupling, synchronization is enhanced at a lower mobility rate than with instantaneous coupling. We argue that these results may be relevant to the synchronization of mobile oscillators in vertebrate segmentation.
\end{abstract}

DOI: 10.1103/PhysRevE.99.062207

\section{INTRODUCTION}

Synchronization of interacting oscillators occurs in many natural and artificial systems, which range from flashing fireflies to coupled pendulums [1-3]. In living systems, the synchronization of oscillators is often essential for biological function. Such is the case of the vertebrate segmentation clock, a biological pattern-formation system based on genetic oscillators [4-6]. This biological clock operates during embryonic development and is responsible for the formation of vertebrate body segments [5]. Proper segment formation relies on the coherence of a collective rhythm produced by the cells that form the clock tissue [4]. Clock malfunction may lead to disease [7], so a relevant question is how these cells self-organize and maintain a collective rhythm.

In zebrafish, it is thought that each cell in the tissue behaves as an autonomous oscillator [8]. Synchronization of singlecell oscillators is achieved through intercellular communication via Notch signaling [9-13]. Communication through Notch signaling is local, as cells need to be close enough to allow for mechanical contact between transmembrane ligands and receptors [14]. Notch signaling involves a cascade of complex events that include synthesis and transport of macromolecules. Such a cascade can introduce communication delays which may alter collective dynamics and patterning [15-20]. Besides this delayed local communication

\footnotetext{
*uriu@staff.kanazawa-u.ac.jp

†morelli@df.uba.ar
}

mechanism, cells move within the posterior zone of the tissue, exchanging neighbors over time [21-26]. This mobility is expected to affect information flow in the tissue and pattern formation [26].

Both communication delays and cell mobility have been separately described in theories of the segmentation clock and shown to produce distinct effects over synchronization dynamics. Theories consisting of coupled oscillators that include explicit delays in the coupling can capture the complexity of Notch communication $[16,17]$. Coupling delays can shift the collective frequency of synchronization, lead to multistability, and affect synchronization dynamics [27-29]. In contrast, theories that describe cellular mobility as a random exchange of neighbors in a lattice revealed that mobility can speed up synchronization with instantaneous coupling [30-32]. Oscillator mobility may promote synchronization by extending the effective range of coupling and coarsening [32-35]. A phase diagram for nearly synchronized mobile oscillators in two dimensions (2D) has been determined from a generic active hydrodynamics theory that describes continuum longwavelength modes [36]. The enhancement of synchronization by mobility may occur even in the presence of gradually recovering intercellular interactions [31] and short-range velocity correlations between cells in the tissue [37]. Aside from vertebrate somitogenesis, it has been shown that faster synchronization also occurs for mobile chaotic oscillators $[38,39]$ and moving integrate and fire oscillators $[40,41]$. The role of mobility as an enhancer of global order in spatially extended systems has also been highlighted in the context of biodiversity [42,43] and social consensus [44]. 
However, little is known about the combined effect of coupling delays and mobility on the synchronization dynamics of coupled oscillators. Coupling delays may significantly change synchronization dynamics of mobile oscillators. Persistent spatially heterogeneous phase patterns, termed chimera states, form near the stability boundaries of in-phase states in the presence of coupling delays and mobility [45]. Formation of such phase patterns slows down the convergence to in-phase states. The presence of coupling delays causes neighboring oscillators to exchange information about their phases at a previous time point. In addition, if there is also mobility, it could happen that these neighboring oscillators were not neighbors at that previous time point. Thus, even outside the regime where chimera states emerge, coupling delays may also influence the effect of mobility on relaxation to the inphase synchronized state.

In this article, we study synchronization dynamics in a theory that brings together coupling delays and mobility. We consider a system of coupled phase oscillators on a onedimensional lattice and show that mobility can speed up synchronization even in the presence of coupling delays. We use both analytical and numerical calculations to describe different dynamical regimes depending on mobility and delays.

\section{MOBILE COUPLED OSCILLATORS WITH DELAYED COUPLING}

Consider a system of $N$ identical phase oscillators placed in a one-dimensional lattice of $N$ sites. Such lattice geometry allows for an analytical calculation of synchronization dynamics as shown below. For simplicity, we choose a lattice spacing of 1 without loss of generality. Each oscillator $i$ with $i=0,1,2, \ldots, N-1$ has a position in the lattice $x_{i}=0,1,2, \ldots, N-1$. Oscillators can move through the lattice, by stochastically exchanging positions with their nearest neighbors. We describe these exchange events as a Poisson process, in which each pair of neighboring oscillators has a probability $\lambda / 2$ of exchanging positions per unit time $[30,32,37]$. In between exchange events, phase dynamics is given by

$$
\frac{d \theta_{i}(t)}{d t}=\omega+\frac{\kappa}{n_{i}} \sum_{j \in V_{i}(t)} \sin \left[\theta_{j}(t-\tau)-\theta_{i}(t)\right]
$$

with

$$
V_{i}(t)=\left\{j \text { such that }\left|x_{j}(t)-x_{i}(t)\right|=1\right\},
$$

where $\omega$ is the autonomous frequency of the oscillators, $\kappa$ is the coupling strength, and $n_{i}$ is the number of neighbors of oscillator $i$. The delay $\tau$ in the coupling function accounts for the time that an oscillator needs to prepare the signal that it will send to its neighbor. In the vertebrate segmentation clock, this delay represents the time that a cell needs to synthesize ligands and export them to the cell membrane $[15,16]$. Because of this coupling delay, oscillators receive information about the phases that their current neighbors had at a previous time. In addition, current neighbors could be different from the ones at that previous time because of mobility.

We use open boundary conditions: The oscillators at both ends of the lattice interact only with their single left or right neighbors respectively and can only exchange positions with them. This choice of open boundary conditions prevents the formation of stable twisted states that may appear for periodic boundary conditions $[33,46]$ and is a better description of the conditions in the segmentation clock tissue.

We multiply Eq. (1) with the timescale $\omega^{-1}$ to render it dimensionless, introducing a dimensionless time $t^{\prime}=\omega t$ and with all parameters in the theory now expressed in terms of this timescale as dimensionless numbers: $\tau^{\prime}=\omega \tau, \kappa^{\prime}=$ $\kappa \omega^{-1}$, and $\lambda^{\prime}=\lambda \omega^{-1}$. In the following, we drop the primes for notational convenience, which amounts to setting $\omega=1$ in Eq. (1).

One way to experimentally examine the underpinnings of the collective organization of the segmentation clock is to perturb coupling with chemical compounds and characterize the dynamics away from the synchronized state $[10,11,18]$. In the absence of coupling, fluctuations can desynchronize the oscillators. After washing out the chemical compound, coupling is restored. The time required to recover synchronization is directly related to the communication mechanism, which in Eq. (1) is described by coupling strength, mobility of oscillators, and coupling delays. Thus, here we are interested in the dynamics of Eq. (1) as it approaches in-phase synchronized solutions. These solutions always exist and have the form

$$
\theta_{i}(t)=\Omega t \quad \forall i
$$

with collective frequency $\Omega$ satisfying [15,27-29,47]

$$
\Omega=1-\kappa \sin (\Omega \tau) \text {. }
$$

For nonmobile oscillators, in-phase synchronized solutions are stable if $[48,49]$

$$
\gamma \equiv \kappa \cos (\Omega \tau)>0 .
$$

Together with Eq. (3), this relation defines multiple stable branches of $\Omega$ values [Fig. 1(a)]. For large values of the coupling delay, there may be more than one stable solution, depending on $\kappa$.

Besides these in-phase solutions, there are antiphase solutions with collective frequency $\tilde{\Omega}=1+\kappa \sin (\tilde{\Omega} \tau)$ which are stable for delay values satisfying $\kappa \cos (\tilde{\Omega} \tau)<0$ [Fig. 1(a)]. In-phase and antiphase solutions can coexist for some values of the coupling delay [shaded regions in Fig. 1(a)]. In the vicinity of these regions, persistent chimera states may form, where ordered and disordered domains coexist [45]. We exclude time-delay values within these regions to avoid the presence of persistent chimera states which affect synchronization dynamics, as has been shown elsewhere [45]. Furthermore, here we fix the value of $\kappa=0.1$ and consider delay values within the first and second stable branches of solutions to avoid in-phase multistability $[15,16]$. We set the number of oscillators $N=100$ throughout the paper.

We solve Eq. (1) using the algorithm previously described in Ref. [45] with an Euler scheme and a time step $\delta t \leqslant 0.0001$, and choose values of the time delay that are multiples of $\delta t$. To reveal synchronization dynamics of mobile oscillators with delayed coupling, we start simulations from random initial phases and examine how these oscillators reach inphase synchronization. With our parameter setting, complete synchronization is always achieved after transient from those initial conditions. 


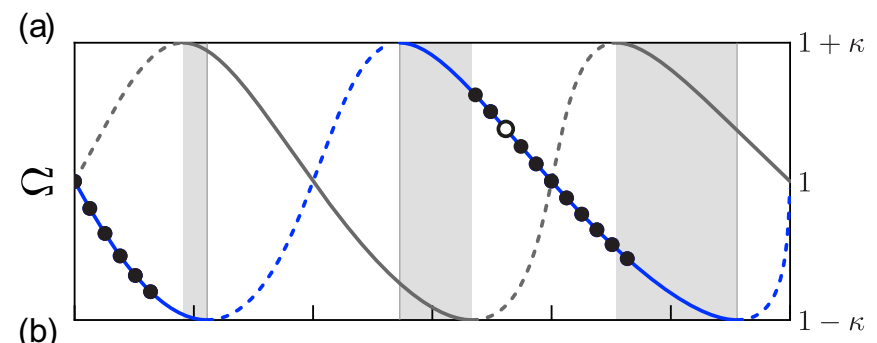

(b)

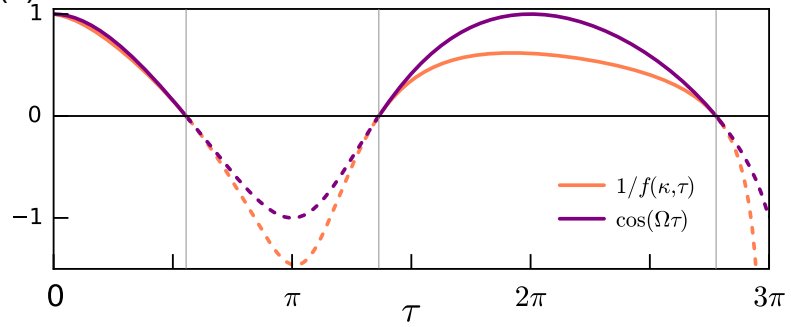

FIG. 1. (a) Parametric plot of the collective frequency for the in-phase synchronized state, Eq. (3) (blue line), together with the analogous collective frequency for the anti-phase solution of Eq. (1) (dark gray line). Solid (dashed) lines indicate solutions are stable (unstable). Dots indicate the sampled values of the time delay used in subsequent figures, in the first and second stable branches of in-phase synchronized solutions. Gray shaded regions indicate overlapping stability of in-phase and antiphase solutions. The open dot marks an approximate value of coupling delay in wild-type zebrafish [16]. (b) The function $1 / f(\kappa, \tau)$ in Eq. (16) that determines the onset of nonlocal behavior (orange), together with $\cos (\Omega \tau)$ that determines the onset of mean-field behavior and stability (purple). Solid lines indicate the regions where in-phase synchronization is stable; dashed lines regions where it is unstable.

\section{SPATIAL PHASE CORRELATIONS}

The effects of mobility on synchronization dynamics of phase oscillators can be captured by spatial phase correlations [32]

$$
\rho_{d}(t)=\left\langle\cos \left[\vartheta_{k+d}(t)-\vartheta_{k}(t)\right]\right\rangle_{k},
$$

where $\vartheta_{k}(t)$ is the phase at site $k$ of the lattice at time $t$. We use $\vartheta_{k}$ instead of $\theta_{i}$ to distinguish phase values at given positions, as opposed to phases of individual oscillators. As oscillators move in the lattice, the value of $\vartheta_{k}$ will correspond to the phase of different oscillators that occupy the lattice site at different times: $\vartheta_{k}(t)=\theta_{i}(t)$ if oscillator $i$ is at site $k$ at time $t$. In Eq. (5), $d$ is the dimensionless distance between lattice sites and $\langle\cdots\rangle_{k}$ indicates the average over sites $k$ with $k=0, \ldots, N-1-d$ (Fig. 2).

Different columns in Fig. 2 correspond to different values of the ratio $\lambda / \kappa$, which measures mobility rate $\lambda$ in terms of the coupling strength $\kappa$. Comparing columns in Fig. 2, we see that synchronization is faster for larger mobility. For zero and low mobility, correlations between close sites increase faster than correlations between distant sites (first two columns in Fig. 2). This difference between short- and longrange correlations indicates that the system forms local order patterns where close sites have similar phases while distant sites do not. For larger values of mobility, all correlations increase simultaneously, indicating that local order patterns do not occur (last column in Fig. 2). Comparing rows in

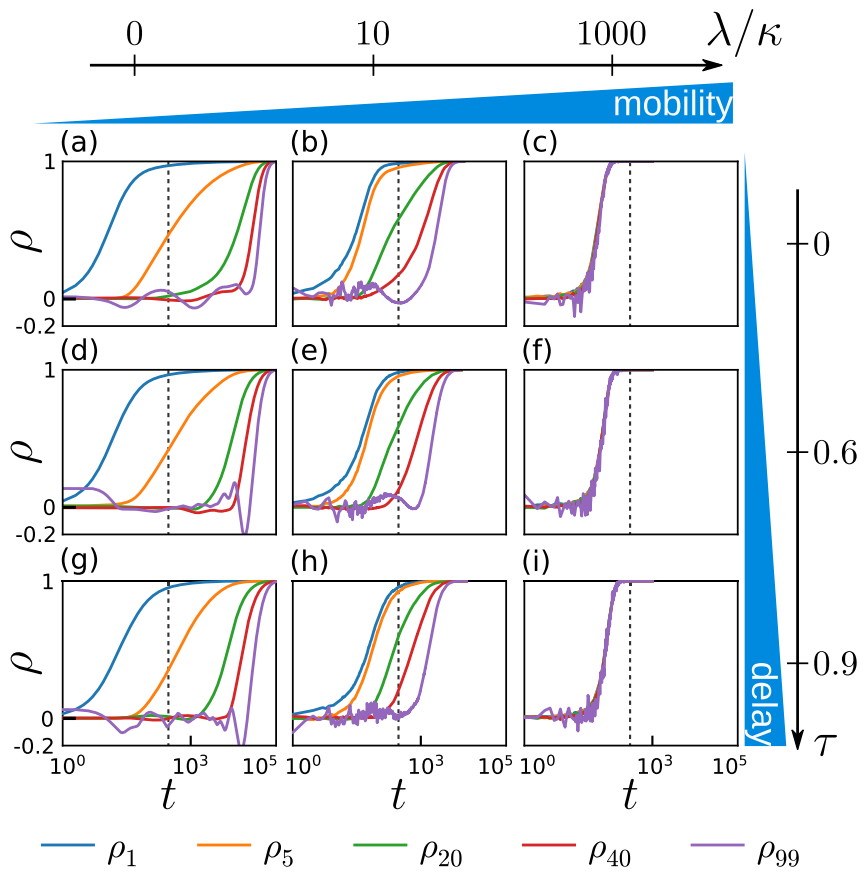

FIG. 2. Time evolution of the spatial phase correlations between lattice sites, for different mobility to coupling ratios $\lambda / \kappa$ (columns) and different delay values (rows). (a) $\lambda / \kappa=0, \tau=0$; (b) $\lambda / \kappa=10$, $\tau=0$; (c) $\lambda / \kappa=1000, \tau=0$; (d) $\lambda / \kappa=0, \tau=0.6$; (e) $\lambda / \kappa=10$, $\tau=0.6$; (f) $\lambda / \kappa=1000, \tau=0.6$; (g) $\lambda / \kappa=0, \tau=0.9$; (h) $\lambda / \kappa=$ $10, \tau=0.9$; and (i) $\lambda / \kappa=1000, \tau=0.9$. A dashed vertical line is drawn at $t=300$ in all panels for reference. In each panel, spatial phase correlations are the average over 192 realizations of initial conditions and mobility.

Fig. 2, we see that this qualitative behavior is observed also with increasing coupling delays. Taken together, these results show that mobility can speed up synchronization even in the presence of coupling delays. Next, we consider the relaxation of spatial modes to quantify the effects of mobility over the time the system needs to reach synchronization.

\section{CHARACTERISTIC RELAXATION TIME}

Relaxation of spatial modes toward complete synchrony can be observed in the behavior of $1-\rho$. After a transient, the system enters an exponential regime $1-\rho \propto e^{-t / T c}$ (Fig. 3). This behavior is observed for all values of the coupling delay studied. To quantify how fast the system approaches complete synchronization, we examine the characteristic time $T_{c}$ in this exponential regime (Fig. 3).

The dependence of characteristic relaxation time $T_{c}$ on the ratio $\lambda / \kappa$ can reveal at which rate mobility starts to influence synchronization dynamics. To determine whether time delays in coupling affect this onset of the effects of mobility, we derive an analytical expression of $T_{c}$ in the presence of both mobility and coupling delays.

The characteristic time $T_{c}$ reflects the relaxation time of the longest spatial mode. For nonmobile oscillators with open boundary conditions, the longest spatial mode has a sinusoidal shape $\sim \cos (\pi x / N)$. In the absence of coupling delays and mobility, the relaxation of this longest mode is determined 


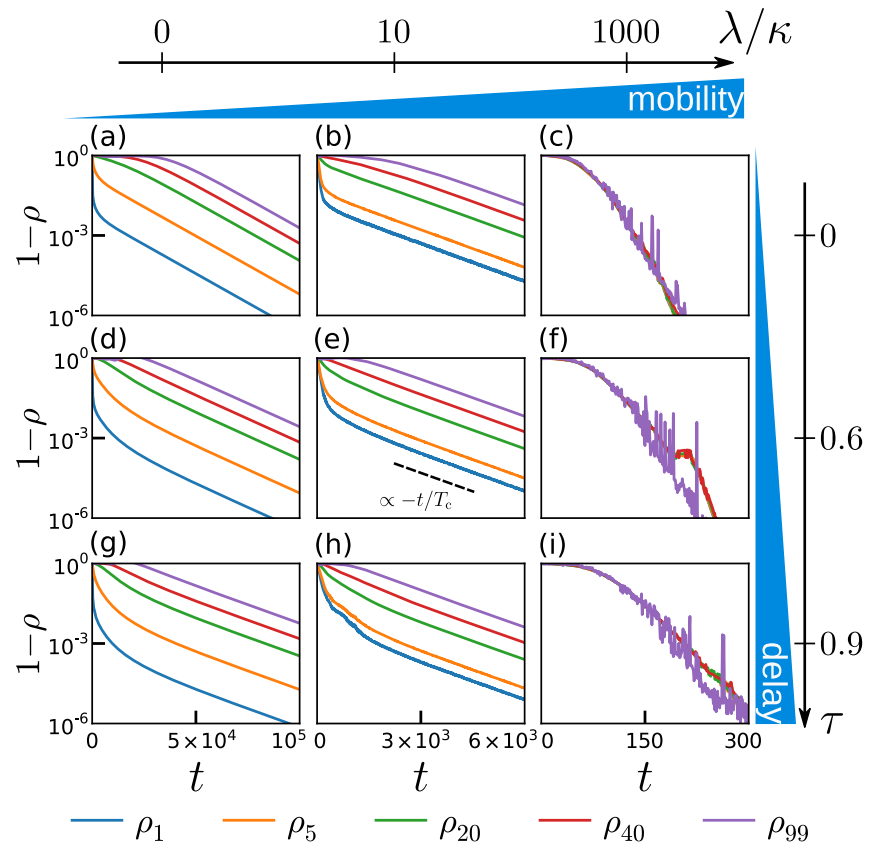

FIG. 3. Exponential relaxation of correlations is revealed in plots of $1-\rho$ vs time. The characteristic time $T_{c}$ is defined as the slope of a linear fit of the long-term exponential regime. Mobility and delay parameters as in Fig. 2.

by the coupling strength and the characteristic time $T_{c}$ can be written as

$$
T_{c}^{-1}=2 \sigma,
$$

where relaxation rate $\sigma$ is a function of the coupling strength. For mobile oscillators, the characteristic time $T_{c}$ includes an additional contribution from mobility

$$
T_{c}^{-1}=2 \sigma+\pi^{2} \lambda / N^{2} .
$$

This expression for $T_{c}$ can be derived considering how a single exchange event affects relaxation of the longest spatial mode in the absence of coupling delays [32]. Derivation of Eq. (7) relies on the fact that the shortest wavelength perturbation induced by the exchange of positions between oscillators relaxes much more quickly than the longest spatial mode.

For instantaneous coupling $\tau=0$, the relaxation rate $\sigma$ depends on the coupling strength and system size as $\sigma \approx$ $\kappa \pi^{2} / 2 N^{2}$ [32]. Substituting this in Eq. (7) gives

$$
T_{c} \approx \frac{N^{2}}{\pi^{2} \kappa} \frac{1}{1+\lambda / \kappa} .
$$

Expression (8) suggests that the onset of the effects of mobility on $T_{c}$ occur for $\lambda / \kappa \sim 1$. These effects are present up to the onset of mean-field behavior at $\lambda / \kappa \sim 2 N^{2} / \pi^{2}$. Above this mean-field onset, each oscillator can effectively interact with every other oscillator in the lattice before its phase changes significantly [32].

Next, we derive an analytical expression for the characteristic time $T_{c}$ for delayed nearest-neighbors coupling. The behavior of spatial phase correlations for mobile oscillators are qualitatively similar among different time delay values (Figs. 2 and 3). Therefore, we conjecture that relaxation of the longest spatial mode is affected by single exchange events in the same way as in the absence of coupling delays, that is, Eq. (7) is still valid. Instead, the relaxation by coupling is modulated by delay. To extend Eq. (8) for delayed coupling, we need to compute the relaxation rate $\sigma$ of the slowest spatial mode for coupled phase oscillators with delayed coupling. In the absence of mobility, the characteristic equation is [29]

$$
\gamma-\sigma=\gamma u e^{\sigma \tau}
$$

where $\gamma=\kappa \cos (\Omega \tau)$ and $u$ is the eigenvalue of the adjacency matrix that indicates the pairs of interacting oscillators. The eigenvector of $u$ corresponds to the longest cosine mode for a one-dimensional (1D) lattice with open boundary, for which $\sigma$ is a real number [29]. We argue that the relaxation rate for the longest spatial mode $|\sigma|$ becomes small for a large system size $N$. For sufficiently small delay $\sigma \tau \ll 1$, we expand the exponential function in Eq. (9) and neglect terms of order $O\left(\sigma^{2} \tau^{2}\right)$

$$
\gamma-\sigma \approx \gamma u(1+\sigma \tau)
$$

Solving this equation with respect to $\sigma$, we obtain the relaxation rate of the longest spatial mode,

$$
\sigma \approx \frac{\gamma(1-u)}{1+\gamma u \tau}
$$

When we substitute expression (11) in (7),

$$
T_{c}=\frac{N^{2}}{\pi^{2}} \frac{1}{\frac{2 N^{2}}{\pi^{2}} \frac{\gamma(1-u)}{1+\gamma u \tau}+\lambda} .
$$

For $N \gg 1$, the eigenvalue can be approximated as $u \approx$ $\cos (\pi / N) \approx 1-\pi^{2} / 2 N^{2}$. When we replace this in Eq. (12) and rearrange terms,

$$
T_{c}=\frac{N^{2}}{\pi^{2} \kappa_{e}} \frac{1}{1+\lambda / \kappa_{e}},
$$

with

$$
\kappa_{e} \equiv \frac{\gamma}{1+\gamma u \tau} .
$$

Equation (13) looks similar to Eq. (8) for nondelayed coupling except that the coupling constant $\kappa$ is replaced by $\kappa_{e}$. Replacing the expressions for $u$ and $\gamma$ in (14) and neglecting $O\left(N^{-2}\right)$ terms,

$$
\kappa_{e} \approx \frac{\kappa \cos (\Omega \tau)}{1+\tau \kappa \cos (\Omega \tau)} .
$$

Thus, delayed coupling alters synchronization dynamics by changing the coupling strength $\kappa$ for an effective coupling strength $\kappa_{e}$, Eq. (15).

Finally, by inserting this expression in (13) and defining

$$
f(\kappa, \tau) \equiv \frac{\kappa}{\kappa_{e}}=\frac{1+\tau \kappa \cos (\Omega \tau)}{\cos (\Omega \tau)},
$$

we arrive at an analytical expression for the characteristic relaxation time in the presence of mobility and coupling delay

$$
T_{c}(\lambda, \tau)=\frac{N^{2}}{\pi^{2} \kappa} \frac{1}{1 / f(\kappa, \tau)+\lambda / \kappa} .
$$

Comparing this result with Eq. (8) explicitly shows that the effect of coupling delays can also be interpreted as a shift in 

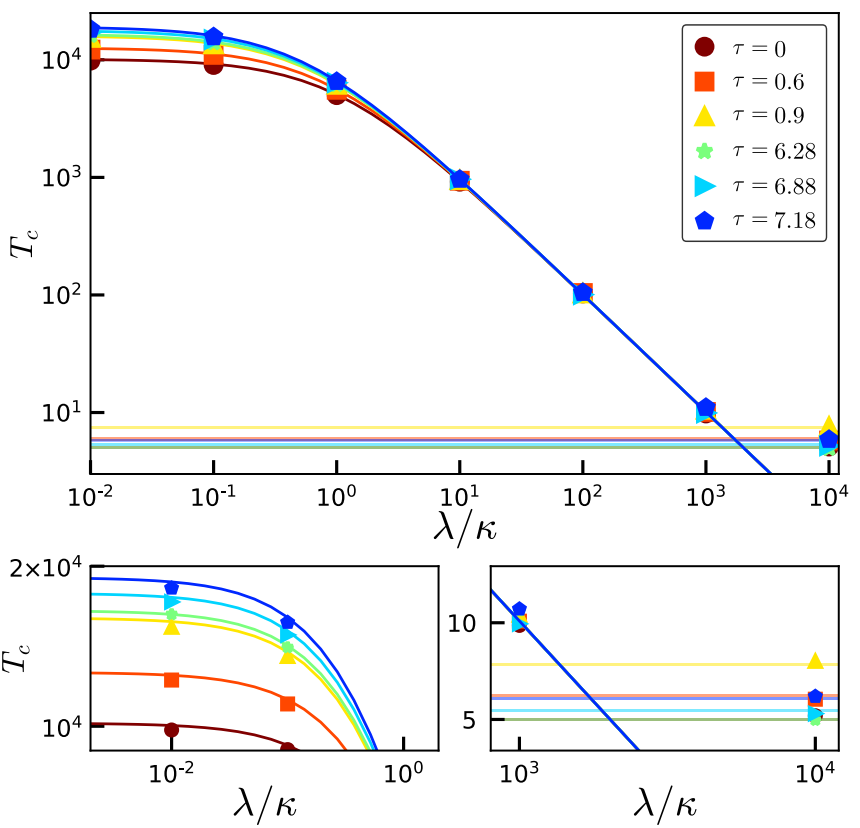

FIG. 4. Characteristic time $T_{c}$ vs mobility to coupling ratio $\lambda / \kappa$, for six different delay values: $\tau=0$ bordeaux, $\tau=0.6$ red, $\tau=0.9$ yellow, $\tau=6.28$ green, $\tau=6.88$ light blue, and $\tau=7.18$ blue. Color markers are numerical simulations and solid lines are theoretical values [Eq. (17)]. Horizontal lines correspond to theoretical values of $T_{c}$ for large mobility in the mean-field regime [Eq. (29)]. Left and right bottom panels display enlargements for small and large mobility, respectively, to emphasize the dependence on delay values. The numerical results were calculated from the averaged spatial correlations over 120 realizations of initial conditions and mobility.

the onset of the effects of mobility determined by $1 / f(\kappa, \tau)$. A plot of $1 / f(\kappa, \tau)$ reveals that for nonvanishing coupling delay the onset of the effects of mobility moves to lower values [Fig. 1(b)]. As we show in Figs. 4 and 5, this analytical result is in good agreement with numerical simulations.

\section{DYNAMICAL REGIMES}

\section{A. Nonmobile regime}

Different dynamical regimes can be identified in Eq. (17) for different mobility to coupling ratios $\lambda / \kappa$. Equation (17) suggests that mobility does not affect synchronization dynamics in a nonmobile regime defined by $\lambda / \kappa \ll 1 / f(\kappa, \tau)$. In contrast, coupling delay strongly affects the relaxation time $T_{c}$ in this regime (bottom left panel in Fig. 4). The relative characteristic time $T_{c}(\lambda, \tau) / T_{c}(\lambda, 0)$ reveals the fold change values of $T_{c}$ as a function of $\tau$ (Fig. 5). For some values of the delay, this fold change in $T_{c}$ is significant (blue in Fig. 5). The analytical result, Eq. (17), is in good agreement with simulations (solid colored lines in Fig. 5). In this nonmobile regime, we can approximate $T_{c}$ by

$$
T_{c}(0, \tau)=\frac{N^{2}}{\pi^{2} \kappa} f(\kappa, \tau)
$$

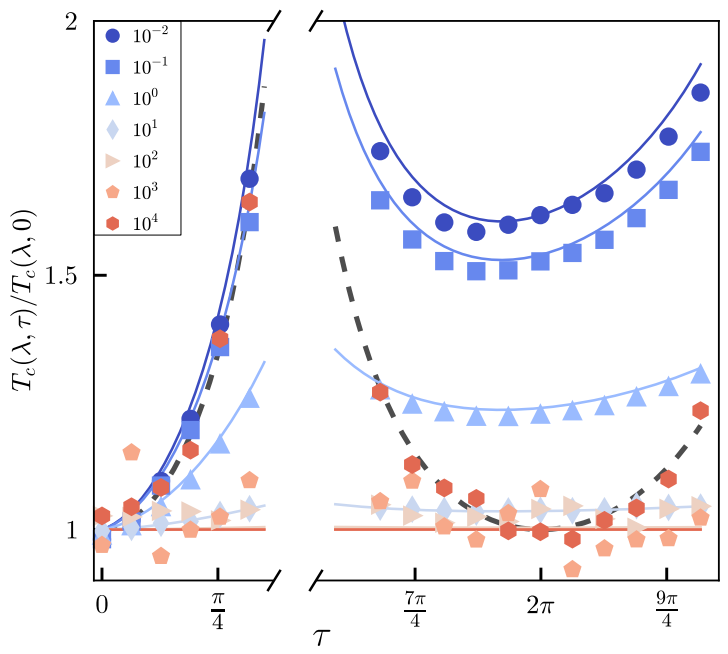

FIG. 5. Characteristic time $T_{c}$ vs delay, for different mobility regimes. Color markers are numerical simulations, solid lines are analytical values from Eq. (17) before the onset of mean-field behavior, and dashed lines are analytical values for mean-field behavior, Eq. (29). Symbols correspond to different values of $\lambda / \kappa$ as indicated in the plot legend.

A rescaling of $T_{c}$ in Eq. (17) by Eq. (18),

$$
\frac{T_{c}(\lambda, \tau)}{T_{c}(0, \tau)}=\frac{1}{1+(\lambda / \kappa) f(\kappa, \tau)},
$$

collapses the curves in Fig. 4 within this regime [Fig. 6(a)].

The effects of mobility become appreciable when

$$
\lambda / \kappa \sim 1 / f(\kappa, \tau)
$$

in Eq. (17), marking the end of the nonmobile regime. The onset of the effects of mobility on synchronization is modulated by the coupling delay through the function $1 / f(\kappa, \tau)$. The mobility rate that can produce a nonlocal behavior depends on the value of the coupling delay, dashed vertical lines in Fig. 6(a). The curves for different delay values, together with the dashed vertical lines that mark the onset of nonlocal behavior, collapse if we plot $T_{c}(\lambda, \tau) / T_{c}(0, \tau)$ as a function (a)

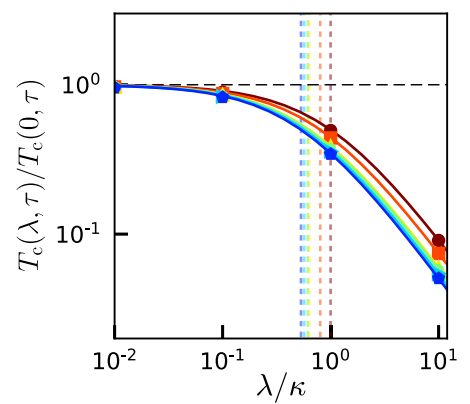

(b)

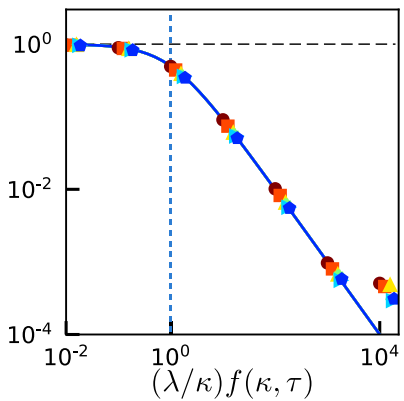

FIG. 6. Onset of mobile regime. (a) Rescaled characteristic time, Eq. (19), as a function of mobility to coupling ratio $\lambda / \kappa$ and (b) as a function of $(\lambda / \kappa) f(\kappa, \tau)$. Dashed vertical lines mark the onset of mobile regime, Eq. (20). Delay values are the same as in Fig. 4: $\tau=$ 0 bordeaux, $\tau=0.6$ red, $\tau=0.9$ yellow, $\tau=6.28$ green, $\tau=6.88$ light blue, and $\tau=7.18$ blue. 
of $(\lambda / \kappa) f(\kappa, \tau)$ [Fig. 6(b)]. These results suggest that in the presence of coupling delays, a slower movement of oscillators could be sufficient to promote synchronization. We come back to this key point in the discussion, to address its relevance for the vertebrate segmentation clock.

\section{B. Mobile regime}

For mobile oscillators with an intermediate mobility rate such that $1 / f(\kappa, \tau)<\lambda / \kappa \ll N^{2}$, relaxation time $T_{c}$ significantly decreases with increasing mobility (Fig. 4). Within this intermediate range, the curves for $T_{c}$ vs $\lambda / \kappa$ for the different delay values collapse, indicating that $T_{c}$ is independent of the delay value in this mobile regime. The ratio $T_{c}(\lambda, \tau) / T_{c}(\lambda, 0)$ decreases and approaches 1 for increasing $\lambda / \kappa$ (Fig. 5). These observations suggest that relaxation of the longest mode is dominated by mobility over delayed coupling in this regime. Taking $\lambda / \kappa \gg 1 / f(\kappa, \tau)$ in Eq. (17),

$$
T_{c} \approx \frac{N^{2}}{\pi^{2}} \frac{1}{\lambda}
$$

that is independent of the delay. Numerical results are consistent with this expectation from the theory; see the light orange symbols in Fig. 5.

\section{Mean-field regime}

The effect of mobility on the characteristic time $T_{c}$ saturates for sufficiently large mobility (bottom right panel of Fig. 4). No spatial mode exists in this regime as reflected in the absence of a distinct correlation length [Figs. 2(c), 2(f), and 2(i)]. Oscillators move so quickly that they effectively behave as a mean-field system, and $T_{c}$ is independent of mobility. However, the characteristic time $T_{c}$ does depend on coupling delays (dark orange hexagons in Fig. 5), indicating that coupling dominates relaxation to complete synchronization again.

An estimation of a mean-field characteristic time $T_{\mathrm{cmf}}$ can be obtained considering a mean-field approximation in which we replace fast mobility with all-to-all coupling

$$
\dot{\theta}_{i}(t)=1+\frac{\kappa}{N} \sum_{j=1}^{N} \sin \left[\theta_{j}(t-\tau)-\theta_{i}(t)\right] .
$$

We introduce a small perturbation $\eta_{i}(t)$ to the synchronized state, $\theta_{i}(t)=\Omega t+\eta_{i}(t)$ with $\left|\eta_{i}(t)\right| \ll 1$. Expanding the sine function around $-\Omega \tau$ and using the expression for the collective frequency,

$$
\Omega=1-\kappa \sin (\Omega \tau),
$$

we can linearize Eq. (22):

$$
\dot{\eta}_{i}(t)=\kappa \cos (\Omega \tau)\left[\frac{1}{N} \sum_{j=1}^{N} \eta_{j}(t-\tau)-\eta_{i}(t)\right] .
$$

For large $N$, we can approximate

$$
\frac{1}{N} \sum_{j=1}^{N} \eta_{j}(t-\tau) \ll\left|\eta_{i}\right| \quad \forall i
$$

and neglect the average perturbation term, so

$$
\dot{\eta}_{i}(t) \approx-\kappa \cos (\Omega \tau) \eta_{i}(t)
$$

(a)

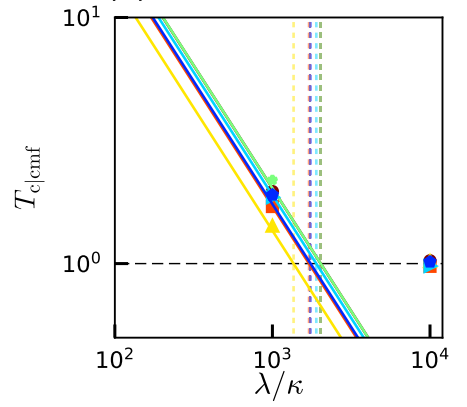

(b)

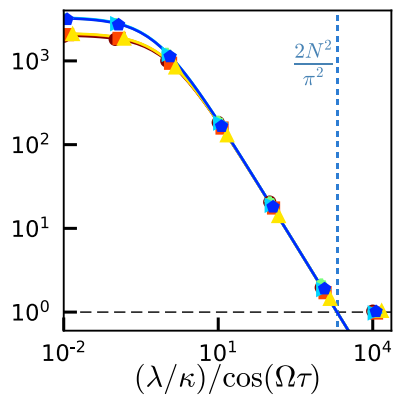

FIG. 7. Onset of mean-field regime. (a) Rescaled characteristic time $T_{c \mid c m f}$ as a function of mobility to coupling ratio $\lambda / \kappa$ and (b) $T_{c \mid c m f}$ as a function of $(\lambda / \kappa) / \cos (\Omega \tau)$. Dashed color lines indicate the onset of mean-field regime for each delay value, Eq. (31). Delay values are the same as in Fig. 4: $\tau=0$ bordeaux, $\tau=0.6$ red, $\tau=$ 0.9 yellow, $\tau=6.28$ green, $\tau=6.88$ light blue, and $\tau=7.18$ blue.

Solutions to Eq. (26) have the form

$$
\eta_{i}(t) \sim e^{-\kappa \cos (\Omega \tau) t} .
$$

Therefore, relaxation of spatial correlations follows

$$
\rho(t) \sim e^{-2 \kappa \cos (\Omega \tau) t},
$$

and we can define a mean-field characteristic time

$$
T_{\mathrm{cmf}}=\frac{1}{2 \kappa \cos (\Omega \tau)} .
$$

This expression is in very good agreement with numerical simulations of mobile oscillators (Figs. 4 and 5), confirming that in this regime oscillators behave as if coupled with all the others.

Rescaling of $T_{c}$ by $T_{\mathrm{cmf}}$

$$
T_{\mathrm{c} \mid \mathrm{cmf}}(\lambda, \tau)=\frac{T_{c}(\lambda, \tau)}{T_{\mathrm{cmf}}}
$$

collapses the data points for different delays in this mean-field regime (Fig. 7).

The onset of mean-field regime is defined by the intersection of the mobile regime described by Eq. (17) and the mean-field regime where $T_{c}=T_{\mathrm{cmf}}$ (Fig. 4). To determine this onset, we evaluate $T_{\mathrm{cmf}}=T_{c}(\lambda, \tau)$, equating Eq. (29) with Eq. (17):

$$
\lambda / \kappa \approx \frac{2 N^{2}}{\pi^{2}} \cos (\Omega \tau) .
$$

Similar to the onset of mobile regime, the onset of mean-field regime is modulated by coupling delays [dashed vertical lines in Fig. 7(a)]. After further rescaling the mobility to coupling ratio according to Eq. (31), the mean-field onset for different delay values collapses [Fig. 7(b)].

The changes in the mean-field onset with the delay values in Eq. (31) parallel the changes in the mobile regime onset $1 / f(\kappa, \tau)$ [Fig. 1(b)]. However, there is a stronger dependence of the mean-field onset on delay compared with the mobile regime onset, despite the similarity between Figs. 6(a) and 7(a) because of logarithmic scale. Equation (31) indicates that the mean-field onset takes the maximum value $2 N^{2} / \pi^{2}$ for the instantaneous coupling $\tau=0$. In addition, the maximum 
value $2 N^{2} / \pi^{2}$ occurs for the specific delay values that satisfy $\Omega \tau=2 \pi n$ with integer $n$. For any other delay values, however, the mean-field onset happens earlier than with instantaneous coupling. Thus, coupling delays can benefit the onset of a mean-field regime where coupling is effectively global.

In summary, the mobility to coupling ratio determines synchronization dynamics of mobile oscillators in the presence of coupling delays. Delays modulate the onset of the different dynamical regimes

$$
\begin{aligned}
& \text { nonmobile } \frac{\lambda}{\kappa} \lesssim \frac{1}{f(\kappa, \tau)} \\
& \text { mobile } \frac{1}{f(\kappa, \tau)}<\frac{\lambda}{\kappa}<\frac{2 N^{2}}{\pi^{2}} \cos (\Omega \tau) \\
& \text { mean-field } \frac{2 N^{2}}{\pi^{2}} \cos (\Omega \tau)<\frac{\lambda}{\kappa} .
\end{aligned}
$$

\section{DISCUSSION}

We have studied synchronization dynamics of mobile oscillators far from the stability boundary of in-phase solution (Fig. 1). In this regime, we found that the effect of mobility on relaxation of the longest spatial mode in the presence of coupling delays was qualitatively similar to that in the absence of delays [32]. Rather, coupling delays change the coupling strength effectively and thereby modulate the onsets of nonlocal and mean-field behaviors [Eq. (32)]. In a complementary previous work, we studied the behavior of mobile oscillators with delayed coupling at the vicinity of stability boundary of in-phase solutions [45]. Near this stability boundary, Fig. 1(a), we observed spatially heterogeneous phase patterns referred to as chimera states. Close to the stability boundary, the eigenvalues of short wavelength modes may become small, resulting in their slow relaxation. How close to this boundary the assumptions that lead to Eq. (7), and consequently to Eq. (32), become invalid remains an open question to be clarified in future work. For chimera states, Eq. (7) is not valid because of the presence of disordered domains.

The segmentation clock features intercellular communication through Notch signaling [9-11,13]. Mutants of the Notch signaling pathway display changes to segmentation period [16]. Furthermore, segmentation clock period can be tuned by gradually changing the strength of Notch signaling with a chemical inhibitor [16]. These experiments are consistent with a delayed coupling theory that describes the dynamics of pattern formation in the segmentation clock $[15,16]$. A fit of the theory to experimental data results in coupling delays that are somewhere within the left part of the second stable branch of solutions [see Fig. 1(a)].

A recent study quantified cell mixing in the zebrafish presomitic mesoderm and tailbud to determine its impact on synchronization of genetic oscillators [25]. A phase oscillator model without coupling delays was adopted to simulate the effect of measured cell mixing on synchronization. Cell mixing reproduced by a physical model enhanced synchronization of phase oscillators in simulations. Our current study reveals that the presence of coupling delays is relevant to the effect of mobility since inclusion of coupling delays shifts the onset of nonlocal behavior to lower mobility values. As discussed in the previous paragraph, the delay value of wild-type zebrafish embryo is estimated to be within the second stable branch. In particular, within this second branch the onset of nonlocal behavior $1 / f(\kappa, \tau)$ is below 0.65 , more than $35 \%$ smaller than in the absence of delays [Fig. 1(b)]. Therefore, given the presence of time delays in Notch signaling [16], our present results further strengthen the possibility that cell mixing influences synchronization in the zebrafish tissue.

Zebrafish segmentation clock cells may behave as autonomous genetic oscillators [8]. Thus, in our current formalism of mobile oscillators, we assume the presence of a stable limit cycle in single cells [50]. In contrast, in the mouse segmentation clock it is thought that single cells behave as excitable systems [51]. Such excitable systems would require a different description for mobile interacting agents, like that of pulse-coupled mobile oscillators [40,41]. It remains an interesting open question how mobility affects synchronization in the mouse segmentation clock, where coupling delays are also present [19,52].

In this work, we analyze the system of phase oscillators with delayed coupling mostly in a linear regime, where oscillators are very close to complete synchronization. We extend previous calculations [32] to include coupling delays and obtain an analytical expression of the characteristic relaxation time in the linear regime. This characteristic relaxation time reveals the onset of nonlocal behavior and that of mean-field regime. Whether these hold in the nonlinear regime, where oscillators are far from complete synchronization, remains an interesting open question. Developing an analytical framework in the nonlinear regime will be an important future challenge.

\section{ACKNOWLEDGMENTS}

L.G.M. acknowledges JSPS Fellowship S17064 and the warm hospitality of Prof. H. Tei's group at Kanazawa University. L.G.M. acknowledges support from ANPCyT PICT 2012 1954, PICT 2013 1301, and FOCEM-Mercosur (COF 03/11). K.U. acknowledges support from JSPS KAKENHI Grants No. 26840085 and No. 17H05762, FY2014 Researcher Exchange Program between JSPS and CONICET, and Kanazawa University Discovery Initiative program. The authors would like to thank Centro de Simulación Computacional para Aplicaciones Tecnológicas (CSC-CONICET) for computational resources.
[1] A. T. Winfree, The Geometry of Biological Time (SpringerVerlag, New York, 1980).

[2] S. C. Manrubia, A. S. Mikhailov, and D. H. Zanette, Emergence of Dynamical Order: Synchronization Phenomena in Complex Systems, 1st ed. (World Scientific, Singapore, 2004).
[3] A. S. Pikovsky, M. G. Rosenblum, and J. Kurths, Synchronization: A Universal Concept in Nonlinear Sciences (Cambridge University Press, Cambridge, UK, 2001).

[4] A. C. Oates, L. G. Morelli, and S. Ares, Development 139, 625 (2012). 
[5] A. Hubaud and O. Pourquié, Nat. Rev. Mol. Cell Biol. 15, 709 (2014).

[6] Y. Harima, I. Imayoshi, H. Shimojo, T. Kobayashi, and R. Kageyama, Semin. Cell Dev. Biol. 34, 85 (2014).

[7] O. Pourquié, Cell 145, 650 (2011).

[8] A. B. Webb, I. M. Lengyel, D. J. Jörg, G. Valentin, F. Jülicher, L. G. Morelli, and A. C. Oates, eLife 5, e08438 (2016).

[9] Y.-J. Jiang, B. L. Aerne, L. Smithers, C. Haddon, D. Ish-Horowicz, and J. Lewis, Nature (London) 408, 475 (2000).

[10] K. Horikawa, K. Ishimatsu, E. Yoshimoto, S. Kondo, and H. Takeda, Nature (London) 441, 719 (2006).

[11] I. H. Riedel-Kruse, C. Müller, and A. C. Oates, Science 317, 1911 (2007).

[12] E. M. Özbudak and J. Lewis, PLoS Genet. 4, e15 (2008).

[13] E. A. Delaune, P. François, N. P. Shih, and S. L. Amacher, Dev. Cell 23, 995 (2012).

[14] R. Kopan and M. X. G. Ilagan, Cell 137, 216 (2009).

[15] L. G. Morelli, S. Ares, L. Herrgen, C. Schröter, F. Jülicher, and A. C. Oates, HFSP J. 3, 55 (2009).

[16] L. Herrgen, S. Ares, L. G. Morelli, C. Schröter, F. Jülicher, and A. C. Oates, Curr. Biol. 20, 1244 (2010).

[17] S. Ares, L. G. Morelli, D. J. Jörg, A. C. Oates, and F. Jülicher, Phys. Rev. Lett. 108, 204101 (2012).

[18] B.-K. Liao, D. J. Jörg, and A. C. Oates, Nat. Commun. 7, 11861 (2016).

[19] H. Shimojo, A. Isomura, T. Ohtsuka, H. Kori, H. Miyachi, and R. Kageyama, Genes Dev. 30, 102 (2016).

[20] D. J. Jörg, L. G. Morelli, and F. Jülicher, Phys. Rev. E 97, 032409 (2018).

[21] A. Lawton, A. Nandi, and M. Stulberg, Development 140, 573 (2013).

[22] B. Bénazéraf, P. Francois, R. E. Baker, N. Denans, C. D. Little, and O. Pourquié, Nature (London) 466, 248 (2010).

[23] M. C. Delfini, J. Dubrulle, P. Malapert, J. Chal, and O. Pourquié, Proc. Natl. Acad. Sci. USA 102, 11343 (2005).

[24] A. Mara, J. Schroeder, C. Chalouni, and S. A. Holley, Nat. Cell Biol. 9, 523 (2007).

[25] K. Uriu, R. Bhavna, A. C. Oates, and L. G. Morelli, Biol. Open 6, 1235 (2017).

[26] K. Uriu and L. G. Morelli, Dev. Growth Differ. 59, 351 (2017).

[27] H. G. Schuster and P. Wagner, Prog. Theor. Phys. 81, 939 (1989).

[28] M. K. S. Yeung and S. H. Strogatz, Phys. Rev. Lett. 82, 648 (1999).
[29] D. J. Jörg, L. G. Morelli, S. Ares, and F. Jülicher, Phys. Rev. Lett. 112, 174101 (2014).

[30] K. Uriu, Y. Morishita, and Y. Iwasa, Proc. Nat. Acad. Sci. USA 107, 4979 (2010).

[31] K. Uriu, S. Ares, A. C. Oates, and L. G. Morelli, Phys. Biol. 9, 036006 (2012).

[32] K. Uriu, S. Ares, A. C. Oates, and L. G. Morelli, Phys. Rev. E 87, 032911 (2013).

[33] F. Peruani, E. M. Nicola, and L. G. Morelli, New. J. Phys. 12, 093029 (2010).

[34] N. Fujiwara, J. Kurths, and A. Díaz-Guilera, Phys. Rev. E 83, 025101(R) (2011).

[35] D. Levis, I. Pagonabarraga, and A. Díaz-Guilera, Phys. Rev. X 7, 011028 (2017).

[36] T. Banerjee and A. Basu, Phys. Rev. E 96, 022201 (2017).

[37] K. Uriu and L. G. Morelli, Biophys. J. 107, 514 (2014).

[38] M. Frasca, A. Buscarino, A. Rizzo, L. Fortuna, and S. Boccaletti, Phys. Rev. Lett. 100, 044102 (2008).

[39] N. Fujiwara, J. Kurths, and A. Díaz-Guilera, Chaos 26, 094824 (2016).

[40] L. Prignano, O. Sagarra, and A. Díaz-Guilera, Phys. Rev. Lett. 110, 114101 (2013).

[41] A. Beardo, L. Prignano, O. Sagarra, and A. Díaz-Guilera, Phys. Rev. E 96, 062306 (2017).

[42] T. Reichenbach, M. Mobilia, and E. Frey, Nature (London) 448, 1046 (2007).

[43] T. Reichenbach and E. Frey, Phys. Rev. Lett. 101, 058102 (2008).

[44] A. Baronchelli and A. Díaz-Guilera, Phys. Rev. E 85, 016113 (2012).

[45] G. Petrungaro, K. Uriu, and L. G. Morelli, Phys. Rev. E 96, 062210 (2017).

[46] D. A. Wiley, S. H. Strogatz, and M. Girvan, Chaos 16, 015103 (2006).

[47] E. Niebur, H. G. Schuster, and D. M. Kammen, Phys. Rev. Lett. 67, 2753 (1991).

[48] Y. Nakamura, F. Tominaga, and T. Munakata, Phys. Rev. E 49, 4849 (1994).

[49] M. G. Earl and S. H. Strogatz, Phys. Rev. E 67, 036204 (2003).

[50] Y. Kuramoto, Chemical Oscillations, Waves, and Turbulence (Springer-Verlag, Berlin, 1984).

[51] A. Hubaud, I. Regev, L. Mahadevan, and O. Pourquie, Cell 171, 668 (2017).

[52] H. Shimojo and R. Kageyama, Semin. Cell Dev. Biol. 49, 76 (2016). 